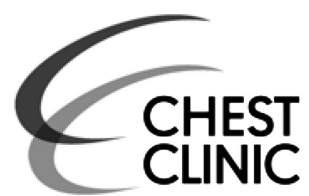

Additional material is published online only. To view please visit the journal online (http://dx.doi.org/10.1136/ thoraxjnl-2014-205249).

Division of

Pharmacoepidemiology and Clinical Pharmacology, Utrecht Institute for Pharmaceutical Sciences, Utrecht University, Utrecht, The Netherlands

\section{Correspondence to}

Dr Aukje K Mantel-Teeuwisse, Division of

Pharmacoepidemiology and Clinical Pharmacology, Utrecht Institute for Pharmaceutical Sciences, Utrecht University, PO Box 80082, 3508 TB Utrecht The Netherlands;

A.K.Mantel@uu.nl

Received 5 February 2014 Accepted 6 February 2014 Published Online First 3 March 2014

\title{
Essential medicines for COPD and asthma in low and middle-income countries
}

\author{
Yaser T Bazargani, Anthonious de Boer, Hubert G M Leufkens, \\ Aukje K Mantel-Teeuwisse
}

\section{ABSTRACT}

Access to medications for chronic disease management is limited in many low and middle-income countries (LMICs), resulting in suboptimal care and avoidable morbidity and mortality. We performed a survey of COPD and asthma medicines that appeared on the national essential medicines lists (NEMLs) of 32 LMICs. Nearly all countries (>90\%) had assigned essential medicines for treatment of exacerbations and early stable disease stages, but not for steps $4(22 \%)$ and $5(6 \%)$ controlled asthma management. The number of treatment options was limited, with long-acting $\beta_{2}$-agonists (LABA) and combination dosage forms being notably absent. Suboptimal availability of chronic respiratory disease medicines suggests that implementation of NEMLs is the main problem in clinical practice.

\section{INTRODUCTION}

Chronic respiratory diseases (CRD) are a major global public health burden. Presently, there are 250 million patients with asthma and 70 million with COPD worldwide. Four million deaths occurred due to CRDs in 2010 globally, of which over $80 \%$ occurred in low and middle-income countries (LMICs) in case of asthma. ${ }^{1}$

These figures raise concerns regarding adequate access to care, including medicines for patients with CRDs across LMICs. Essential medicines are those which satisfy priority healthcare needs of societies. Efforts have been made to improve the availability of essential medicines in LMICs in recent years which has subsequently been reported to reach levels above $75 \%$ in some LMICs.

Selection of appropriate medication for CRDs on national essential medicines lists (NEMLs) is a first step towards achieving adequate access in LMICs, because NEMLs are considered as a basis for public procurement or reimbursement purposes. In this study, we therefore surveyed NEMLs for essential COPD and asthma medicines in LMICs and explored to what extent the choices made would allow treatment of different disease stages of COPD and asthma according to international treatment guidelines.

\section{CrossMark METHODS}

LMICs (according to the World Bank) were eligible for inclusion if their NEML was included in the 'WHO database of essential medicine lists and formularies', and served as a basis for public or private reimbursement or public procurement according to the Pharmaceutical Country Profile survey conducted by WHO in 2011 (see online supplementary annex 1).

Medicines were included if they were categorised as 'medicines acting on the respiratory tract' in the NEML. The medicines were classified according to the Anatomical Therapeutic Chemical (ATC) classification with a distinction being made between short-acting $\beta_{2}$-agonists (SABA), and long-acting $\beta_{2}$-agonists (LABA). Antibiotics, mucolytic agents and oxygen were excluded.

The burden of disease in terms of morbidity was obtained for each country from the Institute for Health Metrics and Evaluation (IHME) database (http://www.healthmetricsandevaluation.org). This is the most recent and reliable source of data on burden of disease which has been frequently cited in global studies. Diseases were ranked in each country based on their burden (year 2010). We used the rank of COPD and asthma in each country as an indicator of their burden relative to other causes of disease burden. Data on geographic regions and income levels were obtained from WHO and the World Bank, respectively.

To identify treatment of different stages of disease, two international treatment guidelines were considered: WHO guideline for COPD management $^{2}$ and the Global Initiative for Asthma (GINA) guideline for asthma management. ${ }^{3}$ Treatments were assigned either to gain and maintain disease control or to manage exacerbation episodes. ATC classes of medicines were considered as unit of analysis in this study.

Descriptive statistics were used to present the results in each category of medicines according to the aforementioned classifications. When the results were compared between different clusters of countries, non-parametric tests were performed to investigate the differences among groups, namely Mann-Whitney U (for comparison between two groups), Kruskal Wallis (for comparison between more than two groups) and $\chi^{2}$ tests (for comparison between categorical variables). All statistical analyses were conducted using SPSS software, V.19.

\section{RESULTS}

Overall, the median number of essential medicines for COPD and asthma on NEMLs in LMICs was 7 (range $=0-22$ ). Over $90 \%$ of the studied countries had selected at least one selective $\beta_{2}$-agonist (all salbutamol), one inhalation corticosteroid (ICS, predominantly beclometasone) and a xanthine derivative (aminophylline and/or theophylline), see online supplementary figure. However, only $10 \%$ 


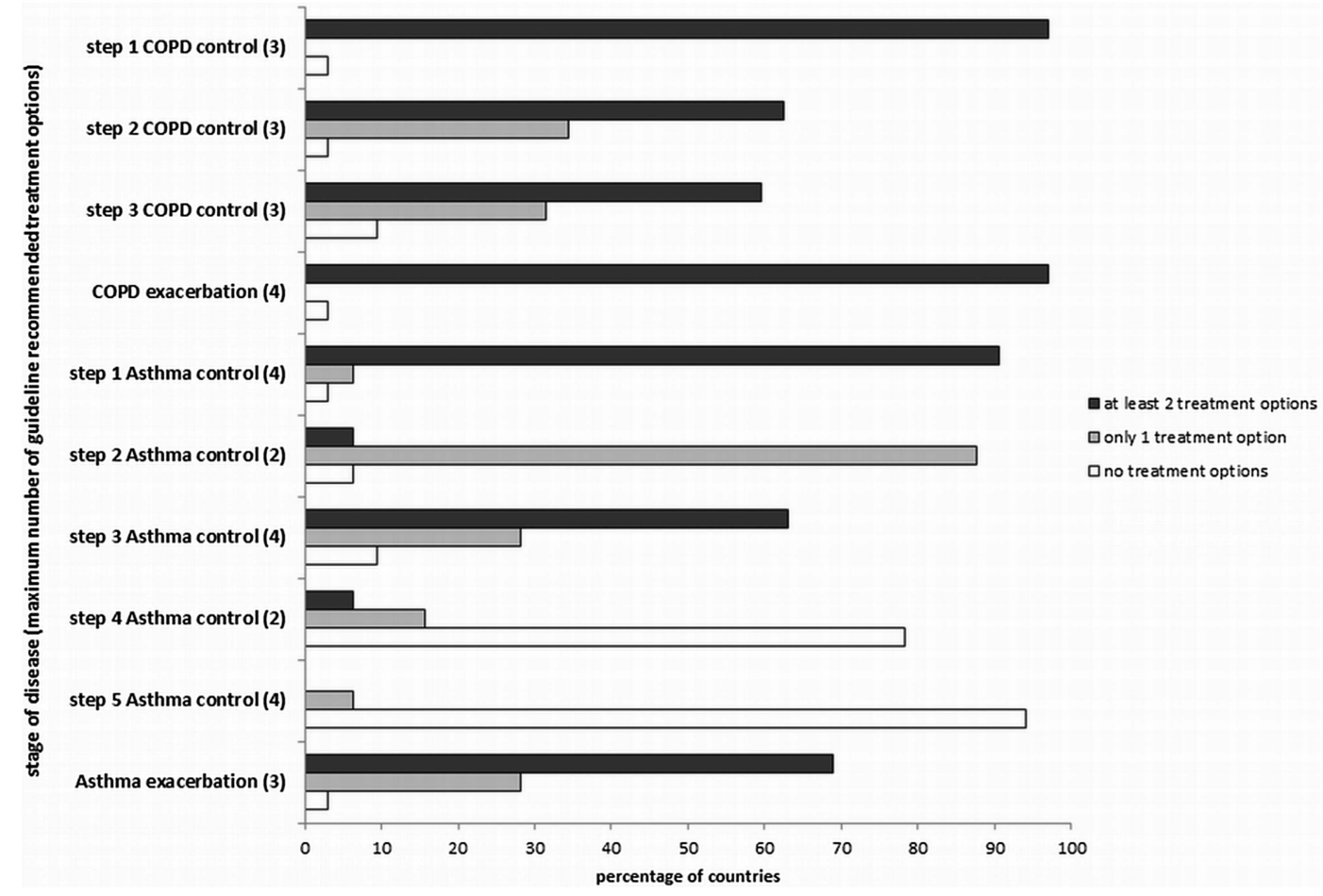

Figure 1 Percentage of countries having treatment options available (according to their NEMLs) for management of different disease stages of COPD and asthma.

\section{Guideline recommendations per disease stage}

Step 1 Asthma control (any reliever); step 2 Asthma control (any reliever+(ICS or LM)); step 3 Asthma control (any reliever+[ICS+(LABA or LM or Xt)] or high-dose ICS); step 4 Asthma control (any reliever+ICS+LABA+(LM or Xt)); step 5 Asthma control (any reliever+step 4 treatments+(OCS or Anti IgE); Asthma exacerbation (SABA+(IAC or ICS or OCS); COPD exacerbation (SABA or ICS or Xt or OCS); step 1 stable COPD (SABA or IAC or Xt); step 2 stable COPD (SABA/LABA+(IAC and/or Xt); step 3 stable COPD (step 2 treatments+ICS).

Reliever: $S A B A$, anticholinergics, short acting theophylline, adrenaline.

ICS, Inhaled Corticosteroids; LM, Leukotriene Modifier; LABA, Long Acting $\beta 2$ agonists; SABA, Short Acting $\beta 2$ agonists Xt, Long acting Xanthine derivative; OCS, Oral Corticosteroids; IAC, Inhaled anticholinergics.

(all upper middle-income) and 22\% of the countries had chosen leukotriene receptor antagonists and $\alpha$ and $\beta$ adrenoreceptor agonists, respectively. Inhaled anticholinergic agents were adopted differently in the NEMLs across income level groups ( $p=0.008$ ) with $20 \%, 61 \%$ and $92 \%$ for the low, lower middle and upper middle-income countries. Thirty percent of the countries studied (all middle-income countries) selected combination dosage forms. Neither low-income countries nor countries in the African region had selected any LABA for their NEML. LABAs were mainly selected by upper middle-income countries (6 out of 8 countries).

Over $90 \%$ of the countries selected essential medicines allowing for management of all treatment steps in COPD and asthma except for steps 4 and 5 of controlled asthma (figure 1). Nearly $80 \%$ of the countries (including all low-income countries) had not selected treatment regimens for the step 4, while only two upper middle-income countries covered step 5 .

The number of treatment options for each step was generally limited. Almost $90 \%$ of countries had incorporated only one treatment option for step 2 management of controlled asthma, while nearly $70 \%$ of the countries had assigned two or less treatment options for step 3. For management of steps 2 and 3 stable COPD, nearly one-third of the countries had included only one treatment option in their NEML (figure 1). In the remaining steps of treatment, predominantly middle-income countries had assigned significantly more treatment options.
A (statistically non-significant) trend towards a higher burden of disease in countries with more treatment options on the NEML was recognised for some stages of the disease management (steps 2 and 4 management of controlled asthma and steps 1 and 2 management of stable COPD, as well as in exacerbations in both conditions).

\section{DISCUSSION}

In most LMICs, essential medicines to treat exacerbations and early stable phases of COPD and asthma were available in NEMLs. However, the majority of these countries had not selected any treatment for step 4 or 5 management of controlled asthma. Additionally, the number of treatment options available in (some) NEMLs for stable COPD, controlled asthma and asthma exacerbation needs further improvement. Differences in selection were seen according to country income level while the burden of disease appeared to have a modest and inconsistent impact. Combination dosage forms and LABAs were mainly incorporated in upper middle-income countries' NEMLs.

Findings of this study may be interpreted as in support of ensuring adequate access to COPD and asthma medicines. However, data on actual availability at health facility level indicates otherwise. Availability of respiratory medicines (either salbutamol $100 \mu \mathrm{g}$ inhaler or beclometasone 250 or $500 \mu \mathrm{g}$ inhaler) was shown to be on average $30.1 \%$ and $43.1 \%$ in the public sector and the private sector of a group of 40 developing 
countries, respectively. ${ }^{4}$ Lack of availability of inhaled corticosteroids (as the most effective controller medication) was repetitively reported in public sector health facilities while it was selected in over $90 \%$ of the NEMLs in our study. In the private sector, the situation is more promising. In a recent study in 50 LMICs, availability of beclometasone and salbutamol inhalers was substantially higher in the private sector compared to the public sector $(41 \%$ and $82 \%$ in the private sector vs $15 \%$ and $54 \%$ in the public sector, respectively). ${ }^{5}$

There are serious affordability concerns for long-term courses of treatment for chronic diseases in the private sector. One year consumption of salbutamol, beclometasone and budesonide bought in the private sector was estimated to cost up to 32 days, 80 days and 800 days of daily minimum wage, respectively. ${ }^{5} \mathrm{By}$ contrast with the low availability shown in these studies, access and usage pattern of CRD medicines in some (upper) middle-income countries has been reported to be comparable with developed countries.

NEMLs are predominantly based on WHO model list of essential medicines, supposedly adjusted for healthcare priorities in different countries. Long-acting medicines and combination dosage forms (ICS plus $\beta_{2}$-agonist) are not included in this model list-despite their clinical advantages-and no application for their inclusion was found as far as the documents could be accessed for the last decade. Inclusion in the WHO model list may encourage LMICs to incorporate these medicines in their NEMLs with a faster pace.

Alarmingly, almost none of the studied LMICs had selected all essential medicines forming the treatment regimen for the 'difficult to treat' asthma patients. The number of treatment options selected for different disease stages is also of concern. Taking the fragility of the supply chain of pharmaceuticals in many LMICs, relying on only one treatment option might seriously threaten patients' health.
Approaches to manage CRDs must be comprehensive and should consist of (a combination of) education, lifestyle improvement, preventive measures and medical treatments. National programmes against respiratory disease have been developed in LMICs with multistakeholder participation. It is worthwhile to study disparities between these national programmes, their action plans and the extent to which their goals have been reached.

According to our study, overall selection of essential medicines in LMICs covers different stages of COPD and asthma treatment (except stages 4 and 5 of controlled asthma). However, sufficient access to the medicines recommended by CRD guidelines is still lacking. Further studies from a health system perspective are needed to determine hurdles which impede access to essential CRD medicines in LMICs despite all the endeavours of functioning healthcare systems.

Contributors YTB: study design, data collection, data analysis, data interpretation and writing. AdB: study design, data analysis, data interpretation and writing. HGML: study concept, data interpretation and final approval. AKM-T: study design, data analysis and verification, data interpretation and writing. YTB (as the principal investigator) and AKM-T (as the corresponding author) guarantee the overall content of the manuscript.

Competing interests None.

Provenance and peer review Not commissioned; internally peer reviewed.

\section{REFERENCES}

1 World health Organization. About chronic respiratory disease. http://www.who.int/ respiratory/about_topic/en/index.html (accessed 28 May 2013).

2 World health Organization. COPD management. 2013. http://www.who.int/ respiratory/copd/management/en/index.html (accessed 01 May 2013).

3 Global initiative for asthma. Global strategy for asthma management and prevention. 4th edn. Vancouver, Canada: GINA, 2013.

4 Cameron A, Roubos I, Ewen $\mathrm{M}$, et al. Differences in the availability of medicines for chronic and acute conditions in the public and private sectors of developing countries. Bull World Health Organ 2011;89:412-21.

5 The International Union Against Tuberculosis and Lung Disease. The Global Asthma Report 2011. Paris, 2011. http://www.globalasthmareport.org/images/files/Global_ Asthma_Report_2011.pdf (accessed 01 May 2013). 